Chirurg 2019 $\cdot 90: 417$

https://doi.org/10.1007/s00104-019-0966-5

Online publiziert: 29. April 2019

(c) Springer Medizin Verlag GmbH, ein Teil von Springer Nature 2019

\section{Originalpublikation}

Baumgart J, Jungmann F, Bartsch F et al (2019) Two-stage hepatectomy and ALPPS for advanced bilateral liver metastases: a tailored approach balancing risk and outcome.

J Gastrointest Surg (Epub ahead of print). https://doi.org/10.1007/s11605-019-04145-9

Einleitung. Die Two-stage-Hepatektomie (TSH) mit oder ohne Portalvenenligatur (PVL) bzw. Portalvenenembolisation (PVE) und das „AssociatingLiver-Partition-and-Portal-Vein-Ligation"(ALPPS)-Verfahren sind chirurgische Strategien in der Behandlung fortgeschrittener Tumoren der Leber. Die aktuelle Forschung beschäftigt sich vor allem mit der Konditionierung der (geschädigten/chemotherapierten) Leber und dem Vergleich zwischen „klassischen" Konditionierungsverfahren und ALPPS.

Die hier vorliegende Arbeit hat das Ziel, die konventionelle TSH mit bzw. ohne PVL/PVE und das ALPPS-Verfahren bei Patienten mit kolorektalen Lebermetastasen $\mathrm{zu}$ beleuchten.

Methodik. Es wurden Daten von Patienten zwischen 2008 und 2017 betrachtet. Neben Komplikationsraten wurden Gesamt- und rezidivfreie Überlebensdaten analysiert.

Ergebnisse. In der vorliegenden Arbeit wurden 790 Leberresektionen bei $611 \mathrm{~Pa}$ tienten analysiert. Nach Ausschluss unilobärer Metastasen $(n=291)$ und klassischer Leberresektionen $(n=257)$ blieben 63 Patienten, die einer TSH zugeführt wurden. Von diesen Patienten abgezogen wurden Patienten, die mittels TSH

F. Dondorf $\cdot M$. Ardelt $\cdot U$. Settmacher

Klinik für Allgemein-, Viszeral- und Gefäßchirurgie, Universitätsklinikum Jena, Jena, Deutschland

\title{
Two-stage-Hepatektomie und ALPPS bei bilateralen Lebermetastasen
}

im Sinne einer Hemihepatektomie links oder einer bilateralen atypischen Resektion versorgt wurden $(n=5)$, sodass letztendlich 58 Patienten blieben, bei denen eine TSH im Sinne einer Hemihepatektomie rechts oder Trisektorektomie erfolgte (16-mal TSH, 20-mal PVL, 14-mal PVE und 8-mal ALPPS-Prozeduren).

In $91 \%$ der Fälle wurde vor der Leberresektion eine Chemotherapie durchgeführt, bei $78 \%$ mit kombinierter Targettherapie.

Der zweite Schritt wurde in $72 \%$ der TSH/PVL/PVE-Gruppe und bei $100 \%$ der ALPPS-Patienten abgeschlossen.

Das mediane Follow-up betrug 15,8 Monate, das mediane Gesamtüberleben 26,7 Monate nach TSH/PVL/PVE und 36,2 Monate nach ALPPS (statistisch nicht signifikant).

Die Rezidivrate lag insgesamt bei $78,7 \%$. Rezidive traten bei $60 \%$ der TSH/PVL/PVE-Kohorte und bei 87,5\% der ALPPS-Kohorte auf (statistisch nicht signifikant). Das mediane rezidivfreie Überleben betrug 5,9 Monate nach TSH/PVL/PVE und 3 Monate nach ALPPS, auch hier ohne statistische Signifikanz.

Diskussion. Die Behandlung bilateraler kolorektaler Lebermetastasen bleibt eine Herausforderung für die hepatobiliäre Chirurgie insbesondere hinsichtlich des tumorfreien Langzeitüberlebens.

Die klassische TSH mit bzw. ohne PVL/PVE hat das Risiko eines „drop out“ zwischen beiden Teilschritten, wohingegen das ALPPS-Verfahren eine höhere Komplettierungsrate mit sich führt. In der Literatur und auch in dieser Arbeit ergibt sich eine höhere Morbidität und Mortalität bei ALPPS im Vergleich zur konventionellen TSH, wobei die geringe Patientenzahl der hier vorliegenden Arbeit die Aussage limitiert.

Im Vergleich zu (konditionierten) erweiterten Leberresektionen ergibt sich bei parenchymschonenden Resektionen zwar ein geringerer Parenchymverlust, der jedoch ein höheres Risiko für R1Resektion aufweist.

Die hohen Rezidivraten und die schnelle Rekurrenz der Tumoren lässt sich unter anderem mit der gewählten Strategie der Autoren erklären, in welcher ALPPS als letztes Mittel betrachtet wird, wohingegen die Literatur hier auch andere Vorgehensweisen betrachtet.

Fazit. Die Konditionierung der funktionellen Restleber vor erweiterter Leberresektion ist eine etablierte Methode. Die präoperative Leberfunktion und die Patientenselektion scheinen jedoch eine sehr relevante Rolle zu spielen. Weitere Arbeiten zu Leberkonditionierung und Regeneration nach Konditionierung sowie der Tumorbiologie sind unumgänglich. Das ALPPS-Verfahren als Erweiterung der hepatobiliären Chirurgie ist weiterhin auf dem Prüfstand aktueller Forschung; hier sind die intensive Analyse der Leberregeneration der Restleber sowie die Tumorbiologie von elementarer Bedeutung.

\section{Korrespondenzadresse}

\section{Dr. med. F. Dondorf}

Klinik für Allgemein-, Viszeral- und Gefäßchirurgie, Universitätsklinikum Jena Am Klinikum 1, 07747 Jena, Deutschland Felix.Dondorf@med.uni-jena.de

Interessenkonflikt. F. Dondorf, M. Ardelt und U. Settmachergeben an, dass kein Interessenkonflikt besteht. 